\section{Are Self-Similar States in Fibonacci Systems Transparent?}

In a recent Letter [1] Maciá and Domínguez-Adame intended to address the physical nature of critical wave functions in a generalized Fibonacci system. Apart from several interesting results presented, one main conclusion reached is that "self-similar wave functions are those exhibiting higher transmission coefficients in a finite Fibonacci system." Although there exist extended or transparent states in many aperiodic systems under some special conditions, the conclusion itself is unfortunately incorrect and misleading, because it is based on a serious miscalculation of the transmission coefficient. In this Comment, we wish to clarify this point and present a correct calculation.

For the Hamiltonian considered in Ref. [1], four original transfer matrices $(X, Y, Z, W)$ can be cast into two new matrices $R_{A}(\equiv Z Y X)$ and $R_{B}(\equiv W X) . \quad R_{A}$ and $R_{B}$ are arranged in a Fibonacci sequence. In the context of the formulation of Ref. [1], there always exists one energy $E$ satisfying a relation

$$
E=\alpha \frac{1+\gamma^{2}}{1-\gamma^{2}},
$$

where $\alpha$ represents the on-site energy, $\gamma$ the transfer integral. For these energies, $\left[R_{A}, R_{B}\right]=0$. Based on
Eq. (1), the global transfer matrix $M(N)$ in Ref. [1] is obtained. Using the $M(N)$, the authors further derive the transmission coefficient as

$$
\tau(N)=\frac{1}{1+\left[\left(1-\gamma^{2}\right)^{2} /\left(4-E^{2}\right) \gamma^{2}\right] \sin ^{2}(N \phi)},
$$

where $\phi$ is a function of $E, \alpha$, and $\gamma$. Then they suggest that the transparent condition be fulfilled when $\sin (N \phi)=0$. However, we must note that the condition $\sin (N \phi)=0$ may not be consistent with Eq. (1), particularly in self-similar states. Therefore, under the condition $\sin (N \phi)=0$ in the self-similar states, Eq. (2) cannot be used and thus $\tau(E)=1$ should not be expected in the self-similar states. In the Letter, they take $N=F_{17}, \quad \gamma=2, \quad \alpha=0.1, \quad$ and $E=-\sqrt{\alpha^{2}+4 \cos (1160 \pi / N)}=0.3348 \ldots \quad$ [correspond to $\sin (N \phi)=0$ ] to plot Fig. 2 in Ref. [1], which statistically exhibits self-similar features and is claimed to be at a transparent state $\tau(E)=1$. Unfortunately, the above three energy parameters $(E, \alpha$, and $\gamma)$ do not satisfy Eq. (1), so their claim that this self-similar state corresponds to a transparent state does not make sense.

Actually, if we consider $E, \alpha$, and $\gamma$ as three independent parameters and denote the global transfer matrix as $T(N)$ with matrix elements $t_{i, j} s((i, j=1,2)$, the transmission coefficient can be obtained as

$$
\tau(N)=\frac{4-E^{2}}{\left[t_{21}-t_{12}+\left(t_{22}-t_{11}\right) E / 2\right]^{2}+\left(t_{22}+t_{11}\right)^{2}\left(1-E^{2} / 4\right)},
$$

where $t_{i, j}$ is a function of the three energy parameters. Note that Eq. (3) is more general and $T_{N} \neq M_{N}$ in a general case. Once $T(N)=M(N)$ (i.e., Eq. (1) holds), it is straightforward to show that Eq. (3) is equivalent to Eq. (2). From Eq. (3), we can check that when Eq. (1) is satisfied, (i) if $\gamma=2, \alpha=0.75$, $E=-1.25$, then $\tau\left(F_{16}\right)=0.5909 \ldots$; (ii) if $\gamma=2$, $\alpha=0.5, \quad E=-5 / 6, \quad$ then $\tau=0.7425 \ldots$, as obtained in Ref. [1]; the corresponding states are not self-similar. However, if we substitute $\gamma=2, \alpha=0.1$, $E=-\sqrt{0.1^{2}+4 \cos ^{2}\left(1160 \pi / F_{17}\right)}$ (corresponding to a self-similar state) into Eq. (3), $\tau(N)=0.2298 \ldots$, instead of the result $\tau=1$ in Ref. [1].

On the other hand, when Eq. (1) is fulfilled, we can further show that $R_{A}=R_{C}^{3}, R_{B}=R_{C}^{2}$, and $M(N)=R_{C}^{N}$, where

$$
R_{C}=\left(\begin{array}{cc}
\gamma^{-1}(E-\alpha) & -\gamma \\
\gamma^{-1} & 0
\end{array}\right)
$$

Then in terms of Chebyshev polynomials of the second order, $M(N)$ can be obtained more easily.

Finally, we wish to pinpoint that if $R_{A}$ and $R_{B}$ commutate, which corresponds to a zero-invariant case, one may find a higher transmission coefficient more easily. For example, (i) $\gamma=2, \alpha=0.1, E=-1 / 6$, $\tau\left(F_{17}\right)=0.9232 \ldots ; \quad$ (ii) $\gamma=1.5, \quad \alpha=0.1, \quad E=$
$-13 / 50, \quad \tau\left(F_{17}\right)=0.9938 \ldots$; (iii) $\gamma=2, \quad \alpha=1.5$, and $E=-2.5, \tau=1$. Notice that these states are not self-similar. It is well known that, in the on-site or in the transfer model, the self-similar states correspond to nonzero invariants [2]. In the mixing model, can the states with zero invariant be self-similar? From both analytical and numerical calculations, we find actually that if $R_{A}$ and $R_{B}$ commutate, there exist only extended states rather than self-similar states, in sharp contrary to the conclusion reached in Ref. [1].

\section{G. J. Jin ${ }^{1}$ and Z.D. Wang ${ }^{2}$}

${ }^{1}$ Department of Physics and National Laboratory of Solid

State Microstructures, Nanjing University

Nanjing 210093, People's Republic of China

${ }^{2}$ Department of Physics, The University of Hong Kong

Pokfulam Road, Hong Kong, People's Republic of China

Received 7 October 1996

[S0031-9007(97)04854-0]

PACS numbers: 71.23.Ft, 61.44. $-\mathrm{n}$

[1] E. Maciá and F. Domínguez-Adame, Phys. Rev. Lett. 76, 2957 (1996).

[2] M. Kohmoto, B. Sutherland, and C. Tang, Phys. Rev. B 35, 1020 (1987). 